\title{
A Simulation Model for Sexual and Vectorial Transmission of Zika Virus (ZIKV)
}

\author{
Oscar Arias Manrique, Dalia M. Muñoz Pizza, Anibal Muñoz Loaiza, Julian A. Olarte García, \\ Carlos A. Abello Muñoz, Steven Raigosa Osorio, Angie Johanna Osorio, Hans Meyer Contreras, \\ John F. Arredondo Montoya
}

Grupo de Modelación Matemática en Epidemiología (GMME), Facultad de Educación, Universidad del Quindío, Quindío, Colombia Email: marcolombia86@hotmail.com

How to cite this paper: Manrique, O.A., Pizza, D.M.M., Loaiza, A.M., García, J.A.O., Muñoz, C.A.A., Osorio, S.R., Osorio, A.J., Contreras, H.M. and Montoya, J.F.A. (2017) A Simulation Model for Sexual and Vectorial Transmission of Zika Virus (ZIKV). Open Journal of Modelling and Simulation, 5, 70-82.

http://dx.doi.org/10.4236/ojmsi.2017.51006

Received: September 7, 2016

Accepted: January 9, 2017

Published: January 12, 2017

Copyright $\odot 2017$ by authors and Scientific Research Publishing Inc. This work is licensed under the Creative Commons Attribution International License (CC BY 4.0).

http://creativecommons.org/licenses/by/4.0/

\begin{abstract}
Nowadays the Zika virus (ZIKV) has been one of the most studied vector-borne diseases due to the considerable outbreaks that have generated around the world as well as due to the new transmission mechanisms and health complications originated. According to statistics of the INS-Colombia for July 2016, $68 \%$ of the population infected by ZIKV (confirmed cases) are pregnant women. Furthermore, the Quindío department belongs to the states with more than $50 \%$ of the total infected persons being pregnant women. Taking into account those characteristics, a theoretical model is proposed and analyzed to describe the population dynamics considering the sexual and vectorial transmission of ZIKV, with special emphasis in the consequences of the non-vectorial transmission in the population. The obtained results with simulations through the beta parameter indicate that the probability of sexual transmission between susceptible women and infected men points out the importance of campaigns to inculcate prevention measures for the safe sexual relationships between ZIKV infected population.
\end{abstract}

\section{Keywords}

ZIKV, Model, Dynamics, Sexual Transmission, Vectorial Transmission

\section{Introduction}

Recently the concern caused by the Zika virus (ZIKV) has increased, and this infection has been turned in a public health problem in several countries of the American continent. This is mainly due to the relationship with diseases such as: microcephaly and Guillain Barre Syndrome [1] [2] [3] and the discovery of new non-vectorial transmission mechanisms, among them, the vertical, sexual and by blood transfusion ways [4].

It is well known that ZIKV is a disease transmitted by the bite of an infected mosquito (principally the Aedes aegypti gender). There are secondary ways of transmission, 
although the knowledge of them is limited. The virus has been detected in human saliva, semen and urine [5] [6] [7]. Also there exist documented cases referent to sexual transmission with clinical evidence and serological tests, in Senegal [8], and afterwards in Tahiti and United Kingdom [9] [10]. More recently evidence has been found in France [6], Italia [11] and United States [12]. Therefore, it is very important to give special attention to the non-vectorial transmission since it is being considered a sexually transmitted disease [13], with repercussions in the outbreaks size and geographical expansion of the virus.

Particularly in Colombia, according to the statistics in the "Boletín Epidemiológico Semanal del Instituto Nacional de Salud" [14], 68\% of ZIKV infected population corresponds to pregnant women. Figure 1 highlighted the territorial extensions of the

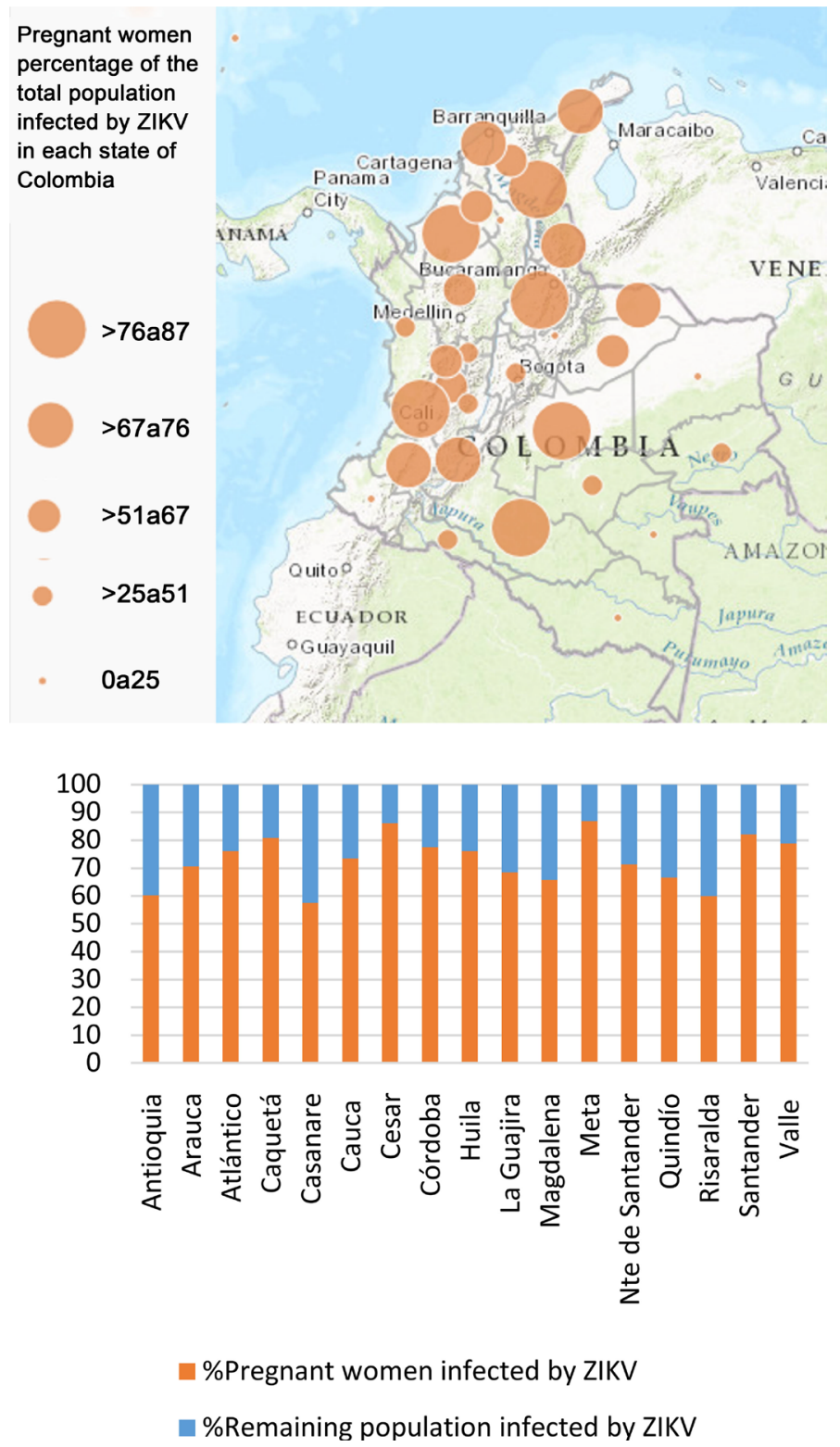

Figure 1. Geospatial distribution of the percentage of pregnant women that are infected by ZIKV in Colombia (upper part). This image has been processed with ArcG is for office software with statistics of the INS [14]. Percentages of the infected pregnant women in each state (lower part). 
country with the highest quantity of infected pregnant women (confirmed cases). We divide those percentages into three main groups, each group (sixteen states) with more than fifty percent of infected expectant women $(58 \%-87 \%)$. Then, it is especially important to study and analyze the sexual transmission in those states. Since the Quindío department is one of the most affected by this issue, we will consider it in our investigation.

Taking into account these facts, we propose a model in $\mathrm{R}^{3}$ which considers the vectorial transmission as well as the sexual transmission of the ZIKV. The basic reproduction number and the stability analysis of the system are obtained. After that, the simulations using the Maple software are carried out to observe the behavior of the epidemic threshold as function of several parameters, the behavior of the sensitivity indexes as well as the transmission probability by sexual way in the human populations. Unlike the model proposed by Gao et al. [15], where it is developed a deterministic model of the vectorial and sexual transmission with statistics for Brazil, Colombia and El Salvador. The parameters used in that model include the population divided in symptomatic and asymptomatic populations on the other hand, our model contemplates the population divided in women and men infected by ZIKV, using the average temperature of Armenia-Quindío, Colombia. Also we focus in parameters which contemplate the susceptible women group, due to the possible complications that arise from the transmission to this fraction of the population [16]. The results of both models support the use of campaigns of sexual protection in the intimate relationships of ZIKV infected persons.

It is important to model this dynamics to offer useful information to the public health care institutions in the country, for the application of preventive measures to the sexual transmission of this virus, this is due to the potential propagation of this disease and the collateral effects that may cause. Note that in Colombia almost all the states present high percentages pregnant women infected by ZIKV (see Figure 1), emphasizing the relevance of the present study.

\section{The Model}

It is proposed a simulation model, in $R^{3}$, based in non-linear ordinary differential equations following the Sir Ronald Ross formalism to describe the dynamics of the sexual and vectorial ZIKV transmission, accounting the following assumptions: closed populations, variations in time of the ZIKV infected pregnant women population, infected men populations, and the virus-carrier $A$. aegypti mosquitoes, and the constant recovery rate of infected women and infected men. Also, it is considered the ZIKV transmission from the infected men to the susceptible women, assuming promiscuity.

The variables of the model are:

$x$ : fraction of women infected by ZIKV at time $t$,

$y$ : fraction of men infected by ZIKV at time $t$ and

$u$ : fraction of virus-carrier mosquitoes at time $t$.

The parameters of the model are shown in Table 1.

The differential equations system to describe the infectious process is (Figure 2):

$$
\frac{\mathrm{d} x(t)}{\mathrm{d} t}=\beta(1-x(t)) y(t)+\eta(1-x(t)) u(t)-(\theta+\mu) x(t)
$$


Table 1. Parameters of the model.

\begin{tabular}{cl}
\hline Parameter & \\
\hline$\beta$ & $\begin{array}{l}\text { Transmission probability by sexual contact between susceptible women and men infected } \\
\text { by ZIKV }\end{array}$ \\
$\eta$ & Transmission probability by the bite of virus-carrier mosquitoes to susceptible women \\
$\theta$ & Recovery rate of infected people \\
$\mu$ & Natural mortality rate of the human population \\
$\xi$ & Transmission probability by the bite of virus-carrier mosquitoes to susceptible men \\
$\sigma$ & Transmission probability to non-carrier mosquitoes by the bite to the infected women \\
$\delta$ & Transmission probability to non-carrier mosquitoes by the bite to the infected men \\
$\epsilon$ & Mortality rate of the mosquitoes \\
\hline
\end{tabular}

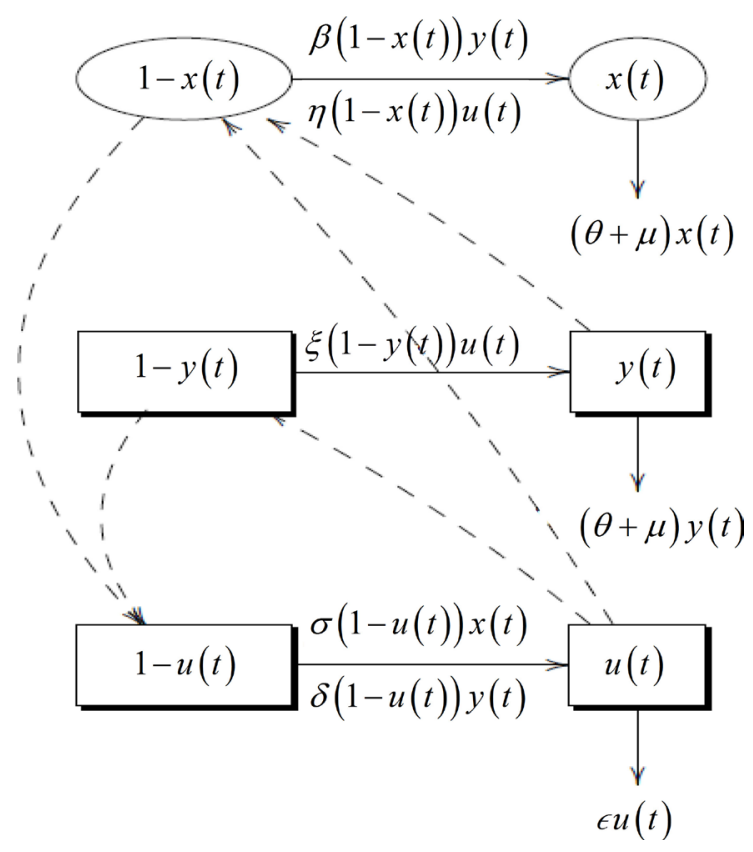

Figure 2. The infectious process diagram.

$$
\begin{gathered}
\frac{\mathrm{d} y(t)}{\mathrm{d} t}=\xi(1-y(t)) u(t)-(\theta+\mu) y(t) \\
\frac{\mathrm{d} u(t)}{\mathrm{d} t}=\sigma(1-u(t)) x(t)+\delta(1-u(t)) y(t)-\epsilon u(t)
\end{gathered}
$$

where $\theta, \mu, \epsilon>0,0<\beta, \eta, \xi, \sigma, \delta<1$, with initial conditions $x(0)=x_{0}, y(0)=y_{0}$ and $u(0)=u_{0}$.

And, the region where the system trajectories have epidemiological sense is,

$$
\Phi=\left\{(x, y, z) \in R_{+}^{3}: 0 \leq x \leq 1,0 \leq y \leq 1,0 \leq u \leq 1\right\}
$$

\section{Stability Analysis}

The stability analysis begins calculating the equilibrium points by solving the algebraic system, which is associated to the non-linear differential equations, $\frac{\mathrm{d} x(t)}{\mathrm{d} t}=0$, 


$$
\begin{aligned}
& \frac{\mathrm{d} y(t)}{\mathrm{d} t}=0 \text { and } \frac{\mathrm{d} u(t)}{\mathrm{d} t}=0 ; \\
& \begin{aligned}
& 0=\beta(1-x(t)) y(t)+\eta(1-x(t)) u(t)-(\theta+\mu) x(t) \\
& \quad 0=\xi(1-y(t)) u(t)-(\theta+\mu) y(t) \\
& 0= \sigma(1-u(t)) x(t)+\delta(1-u(t)) y(t)-\epsilon u(t)
\end{aligned}
\end{aligned}
$$

Obtaining $y(t)$ from Equation (5), it is obtained the following equation,

$$
y(t)=\frac{\xi u(t)}{(\theta+\mu+\xi u(t))}
$$

Substituting (7) in (4), it is found:

$$
\beta(1-x(t)) \frac{\xi u(t)}{(\theta+\mu+\xi u(t))}+\eta u(t)+\eta x(t) u(t)=(\theta+\mu) x(t)
$$

Simplifying the previous equation, we have,

$$
x(t)=\frac{u(t)(\beta \xi+\eta(\theta+\mu+\xi u(t)))}{\beta \xi u(t)+(\theta+\mu+\eta u(t))(\theta+\mu+\xi u(t))}
$$

Replacing (7) and (8) in (6), we obtain:

$$
u(t)\left\{\frac{\sigma(1-u(t))(\beta \xi+\eta(\theta+\mu+\xi u(t)))}{\beta \xi u(t)+(\theta+\mu+\eta u(t))(\theta+\mu+\xi u(t))}+\frac{\delta(1-u(t)) \xi}{(\theta+\mu+\xi u(t))}-\epsilon\right\}=0
$$

where $u(t)=0$;

$$
\frac{\sigma(1-u((t))(\beta \xi+\eta(\theta+\mu+\xi u(t)))}{\beta \xi u(t)+(\theta+\mu+\eta u(t))(\theta+\mu+\xi u(t))}+\frac{\delta(1-u(t)) \xi}{(\theta+\mu+\xi u(t))}=\epsilon
$$

For $u(t)=0$, it is obtained $y(t)=0$ and $x(t)=0$; then we have the free of infection equilibrium point, $E_{0}=(0,0,0)$.

Simplifying Equation (10), it is derived the following equation,

$$
A u^{3}+B u^{3}+C u+D=0
$$

where,

$$
\begin{aligned}
A= & \xi^{2} \sigma \eta+\delta^{3} \xi^{3} \eta+\epsilon \eta \xi^{2} \\
B= & \sigma \eta \xi(\theta+\mu)-\xi[\sigma \eta \xi-\sigma(\beta \xi+\eta(\theta+\mu))] \\
& +\delta^{2} \xi^{2}[\xi(\beta+\theta+\mu)+\eta(\theta+\mu)]+\epsilon \xi[(\theta+\mu)(2 \eta+\xi)+\xi \beta] \\
C= & -\sigma \xi[\beta \xi+\eta(\theta+\mu)]-[\sigma \eta \xi-\sigma[\beta \xi+\eta(\theta+\mu)]](\theta+\mu) \\
& +\left[\delta^{2} \xi^{2}(\theta+\mu)^{2}\right]+\epsilon(\theta+\mu)[(\theta+\mu)(2 \xi+\eta)+\xi \beta] \\
D= & -\sigma[\beta \xi+\eta(\theta+\mu)](\theta+\mu)+\epsilon(\theta+\mu)^{3}
\end{aligned}
$$

Due to $A>0$ and if we assume that at least one of the $B, C$ and $D$ is less than zero, it guarantees a change of sign in the coefficients of the quadratic equation. Following the signs rule of Descartes it is obtained a positive and real root $(u)$, then, we have an infection prevalence equilibrium point, $E_{1}=(\hat{x}, \hat{y}, \hat{u})$, 


$$
\begin{gathered}
\hat{x}(t)=\frac{\hat{u}(t)(\beta \xi+\eta(\theta+\mu+\xi \hat{u}(t)))}{\beta \xi \hat{u}(t)+(\theta+\mu+\eta \hat{u}(t))(\theta+\mu+\xi \hat{u}(t))} \\
\hat{y}=\frac{\xi \hat{u}(t)}{\theta+\mu+\xi \hat{u}(t)}
\end{gathered}
$$

In the linearization process for the non-lineal equations system (1)-(3), is calculated the Jacobian matrix at the generic equilibrium point $E=(\tilde{x}, \tilde{y}, \tilde{u})$,

$$
J(E)=\left(\begin{array}{ccc}
-(\beta \tilde{y}+\eta \tilde{u}+\mu+\theta) & \beta(1-\tilde{x}) & \eta(1-\tilde{x}) \\
0 & -(\tilde{u} \xi+\mu+\theta) & \xi(1-\tilde{y}) \\
\sigma(1-\tilde{u}) & \delta(1-\tilde{u}) & -(\delta \tilde{y}+\sigma \tilde{x}+\epsilon)
\end{array}\right)
$$

Evaluating the Jacobian matrix in the free of infection equilibrium point, we obtain:

$$
J\left(E_{0}\right)=\left(\begin{array}{ccc}
-(\mu+\theta) & \beta & \eta \\
0 & -(\mu+\theta) & \xi \\
\sigma & \delta & \epsilon
\end{array}\right)
$$

So, the characteristic equation $\left|J\left(E_{0}\right)-\lambda I\right|=0$ is,

$$
\lambda^{3}+a_{1} \lambda^{2}+a_{2} \lambda+a_{3}=0
$$

where,

$$
\begin{aligned}
& a_{1}=2(\mu+\phi)+\epsilon \\
& a_{2}=(\mu+\phi)(\mu+\phi+2 \epsilon)+\xi \delta-\sigma \eta \\
& a_{3}=1-R_{0}
\end{aligned}
$$

Under the Routh-Hurwitz criterion, it should be accomplished that $a_{1}>0, a_{3}>0$ and $a_{1} a_{2}>a_{3}$. As a consequence the equilibrium point $E_{0}$ is local and asymptotically stable.

At the equilibrium point $E_{1}$, the characteristic equation $\left|J\left(E_{1}\right)-\lambda I\right|=0$ corresponding to the Jacobian matrix $J\left(E_{1}\right)$ is;

$$
\operatorname{det}\left(\begin{array}{ccc}
-(a+\lambda) & \beta(1-\hat{x}) & \eta(1-\hat{x}) \\
0 & -(b+\lambda) & \xi(1-\hat{y}) \\
\sigma(1-\hat{u}) & \delta(1-\hat{u}) & -(c+\lambda)
\end{array}\right)=0
$$

where, $a=\beta \hat{y}+\eta \hat{u}+\mu+\theta, \quad b=\xi \hat{u}+\mu+\theta$ and $c=\delta \hat{y}+\eta \sigma+\epsilon$.

Solving by the first column and cofactors, we have,

$$
\lambda^{3}+a_{1} \lambda^{2}+a_{2} \lambda+a_{3}=0
$$

with

$$
\begin{aligned}
& a_{1}=a+b+c \\
& a_{2}=a(b+c)+b c-\delta \xi(1-\hat{u})(1-\hat{y}) \\
& a_{3}=a b c-a \delta \xi(1-\hat{u})(1-\hat{y})-\sigma(1-\hat{u}) \beta \xi(1-\hat{x})(1-\hat{y})-\sigma \eta(1-\hat{u})(1-\hat{x}) b
\end{aligned}
$$

Applying the Routh-Hurwitz criterion, this equation has 3 roots with negative real part if the inequalities are met $a_{1}>0, a_{3}>0$ and $a_{1} a_{2}>a_{3}$, as consequence the equilibrium point $E_{1}$ is local and asymptotically stable, if not, the point is unstable. 


\section{Basic Reproduction Number $R_{0}$}

The so called epidemic threshold $R_{0}$, basic reproduction number, indicates the average number of cases that an infectious person produces during the average time of the infection in a susceptible population [17] [18], this threshold is determined assuming that, at least one of the populations, is fully susceptible. Such fractions are approximated taking into account the presence of the Zika virus, as $1-x \approx 1,1-y \approx 1$ and $1-u \approx 1$.

Then doing $y$ in function of $u$, from Equation (2) is obtained $y=\frac{\xi}{\theta+\mu} u$. Replacing this value in Equation (1), then we have $x=\frac{\beta \xi}{(\theta+\mu)^{2}}+\frac{\eta}{\theta+\mu}$. Also, $u$ increases $(u>0)$ when $\frac{\mathrm{d} u}{\mathrm{~d} t}>0$ :

$$
\left(\frac{\xi \sigma \beta}{(\theta+\mu)^{2}}+\frac{\sigma \eta}{\theta+\mu}+\frac{\delta \xi}{\theta+\mu}-\varepsilon\right) u>0
$$

so,

$$
R_{0}=\frac{\eta \sigma}{\varepsilon(\theta+\mu)}+\frac{\xi \delta}{\varepsilon(\theta+\mu)}+\frac{\xi \beta \sigma}{\varepsilon(\theta+\mu)^{2}}=R_{0}^{1}+R_{0}^{2}+R_{0}^{3}
$$

where;

$R_{0}^{1}$ is the threshold corresponding to the virus-carrier mosquitoes and infected pregnant women.

$R_{0}^{2}$ is the threshold corresponding to the virus-carrier mosquitoes and infected men.

$R_{0}^{3}$ is the threshold corresponding to the sexual and vectorial transmission.

For a best understanding of the basic reproduction number $R_{0}$, the additive and multiplicative effects of $R_{0}$ indicate that the vector would be able to transmit the virus to susceptible women and men, the women can acquire the virus by sexual contact, also the vector may get the virus from an infected person.

The terms $\frac{\eta}{\epsilon}$ and $\frac{\xi}{\epsilon}$ indicate the incidence (new cases of ZIKV) in the female and male susceptible populations, respectively, during the lifetime of the vector. These populations generate the quantities $\frac{\sigma}{\theta+\mu}$ and $\frac{\delta}{\theta+\mu}$ of virus-carrier mosquitoes.

The term $\frac{\xi}{\epsilon} \frac{\beta}{\theta+\mu}$ indicates the incidence in the population of susceptible women due to sexual contact between a previously infected man (which gets infected by the bite of a mosquito) and a healthy woman, during a short infectious period of the mosquito or human. This results in a number $\frac{\sigma}{\theta+\mu}$ of infected women.

\section{Numerical Analysis of Stability}

For an average annual temperature in Armenia-Quindío, Colombia of $19.5^{\circ} \mathrm{C}$, were estimated the transmission probabilities $\xi, \sigma$ and $\epsilon$, using the functions contained in 
[18] [19],

$$
\begin{gathered}
\xi=0.0729 T-0.9037,12.4^{\circ} \mathrm{C} \leq T \leq 26.1^{\circ} \mathrm{C} \\
\sigma=0.001044 T(T-12.286)(32.461-T)^{\frac{1}{2}}, 12-4^{\circ} \mathrm{C} \leq T \leq 26.1^{\circ} \mathrm{C} \\
\epsilon=0.8692-0.1590 T+0.01116 T^{2}-0.0003408 T^{3}+0.000003809 T^{4}
\end{gathered}
$$

where $10.54^{\circ} \mathrm{C} \leq T \leq 33.4^{\circ} \mathrm{C}$, with a life expectancy in Colombia of 75 years, $\mu=\frac{1}{75 \times 365}$, an average period of transmissibility $E\left[x_{2}\right]$ of 7 days and considering the Poission theory of infectious process in epidemiology $E\left[x_{2}\right]=\frac{1}{\theta+\mu}$ the parameter $\theta$ was estimated; the value of the parameter $\delta$ and $\eta$ are assigned according the literature [16], as described in the Table 2.

The local stability analysis is carried out using the estimated parameters. We start with the calculation of the free of infection and prevalence equilibrium points, which are determined doing the differentiation of the system (1)-(3) equal to zero, and solving the non-linear algebraic system for each demographic variable.

$$
\begin{gathered}
\beta(1-x) y+(0.5178)(1-x) u-(0.14+0.0003) x=0 \\
(0.5178)(1-y) u-(0.14+0.0003) y=0 \\
(0.52872)(1-u) x+(0.52872)(1-u) y-(0.03604) u=0
\end{gathered}
$$

Using the MAPLE software the equilibrium points are determined.

- For $\beta=0.3: E_{0}=(0,0,0), E_{1}=(0.838,0.779,0.959)$

- For $\beta=0.6: E_{0}=(0,0,0), E_{1}=(0.873,0.779,0.960)$

- For $\beta=0.8: E_{0}=(0,0,0), E_{1}=(0.888,0.780,0.960)$

With information in the Table 2 and the calculated equilibrium points, we obtain the local sensitivity analysis. Also, using the Jacobian matrix the results of Table 3 are obtained.

\section{Local Sensitivity Analysis}

Another threshold is obtained using the local sensitivity analysis, this one represents a relative measure of the change in one variable when the value of one parameter changes

Table 2. Estimated parameters for the model.

\begin{tabular}{ccccccccc}
\hline Parameter & $\eta$ & $\theta$ & $\mu$ & $\xi$ & $\sigma$ & $\delta$ & $\varepsilon$ & $\beta$ \\
\hline Value & 0.5178 & 0.14 & 0.0003 & 0.5178 & 0.52872 & 0.52872 & 0.03604 & $0.3-0.6-0.8$ \\
\hline
\end{tabular}

Table 3. Local stability analysis for each $\beta$.

\begin{tabular}{cccc}
\hline$\beta$ & Equilibrium point & Eigenvalues & Stability \\
\hline 0.3 & $(0,0,0)$ & $0.7157,-0.3032,-0.7290$ & Unstable \\
0.3 & $(0.838,0.779,0.959)$ & $-0.6254,-0.8988,1.2636$ & Stable \\
0.6 & $(0,0,0)$ & $0.7669,-0.5418,-0.5418$ & Unstable \\
0.6 & $(0.873,0.779,0.960)$ & $-0.6273,-0.9166,-1.2634$ & Stable \\
0.8 & $(0,0,0)$ & $0.7971,-0.5569,-0.5569$ & Stable \\
0.8 & $(0.888,0.780,0.960)$ & $-0.6278,-0.9249,-1.2639$ & Stable \\
\hline
\end{tabular}


[20] [21] [22]. The sensitivity index for $R_{0}$, is calculated with the following expression,

$$
I_{p}^{R_{o}}=\frac{\partial R_{o}}{\partial p} \times \frac{p}{R_{0}}
$$

where, $p$ is a parameter. So, the next index have been obtained.

$$
\begin{gathered}
I_{\xi}^{R_{o}}=\frac{(\delta(\theta+\mu)+\beta \sigma) \xi}{\xi(\theta+\mu) \delta+\sigma(\beta \xi+\eta(\theta+\mu))} \\
I_{\delta}^{R_{o}}=\frac{\xi(\theta+\mu) \delta}{(\delta(\theta+\mu)+\beta \sigma) \xi+(\theta+\mu) \eta \sigma} \\
I_{\eta}^{R_{o}}=\frac{(\theta+\mu) \eta \sigma}{\xi(\theta+\mu) \delta+\sigma(\beta \xi+\eta(\theta+\mu))} \\
I_{\sigma}^{R_{o}}=\frac{\sigma(\beta \xi+\eta(\theta+\mu))}{\xi(\theta+\mu) \delta+\sigma(\beta \xi+\eta(\theta+\mu))} \\
I_{\theta}^{R_{o}}=\frac{-((2 \beta \xi+\eta \mu+\eta \theta) \sigma+\xi(\theta+\mu) \delta) \theta}{(\theta+\mu)(\sigma(\beta \xi+\eta \mu+\eta \theta)+\xi(\theta+\mu) \delta)} \\
I_{\mu}^{R_{o}}=\frac{-((2 \beta \xi+\eta \mu+\eta \theta) \sigma+\xi(\theta+\mu) \delta) \mu}{(\theta+\mu)(\sigma(\beta \xi+\eta \mu+\eta \theta)+\xi(\theta+\mu) \delta)} \\
I_{\beta}^{R_{o}}=\frac{-\beta \sigma \xi}{\xi(\theta+\mu) \delta+\sigma(\beta \xi+\eta(\theta+\mu))}
\end{gathered}
$$

The results of the sensitivity analysis of $R_{0}$ respect to each parameter are shown in Table 4.

In Figure 3, it is presented the behavior of the basic reproduction number respecting Table 4. Sensitivity index of $R_{0}$ respecting each parameter.

\begin{tabular}{ccccccccc}
\hline Parameter & $\xi$ & $\delta$ & $\eta$ & $\sigma$ & $\epsilon$ & $\theta$ & $\mu$ & $\beta$ \\
\hline$I_{p}^{R_{0}}$ & 0.758 & 0.241 & 0.241 & 0.758 & -1 & -1.513 & -0.003 & 0.5167
\end{tabular}
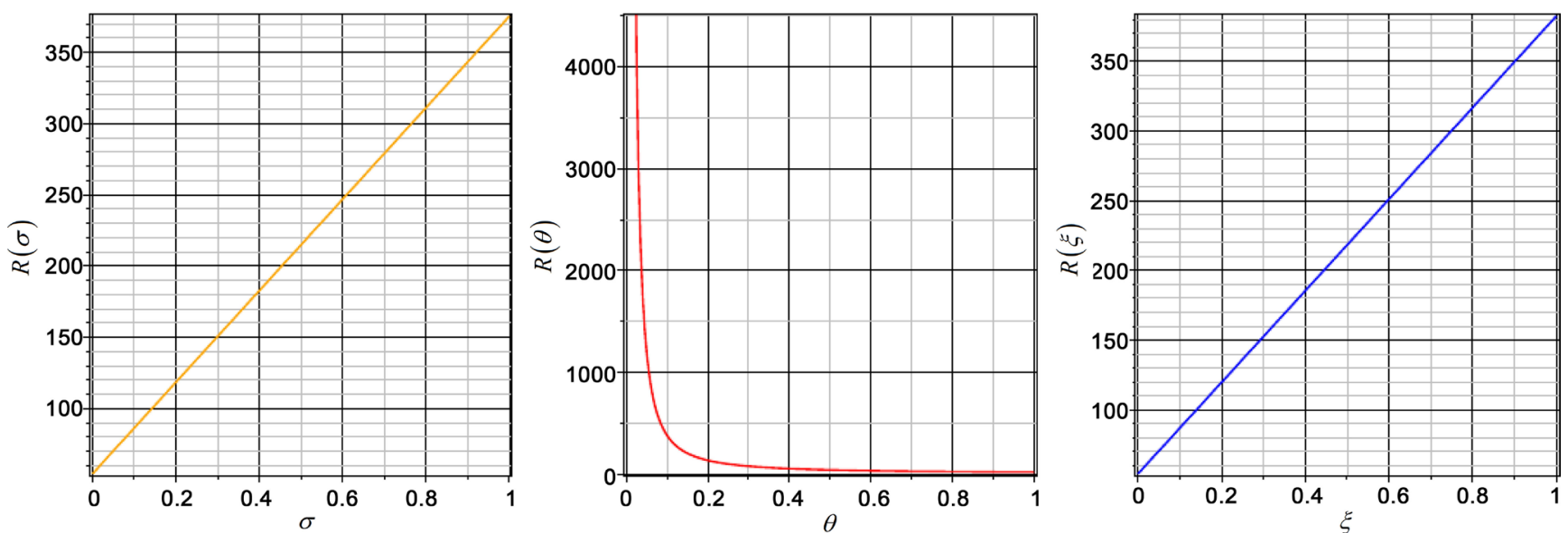

Figure 3. Behavior of the threshold ( $\left.R_{0}\right)$ respecting to $\sigma, \theta$, and $\xi$. 

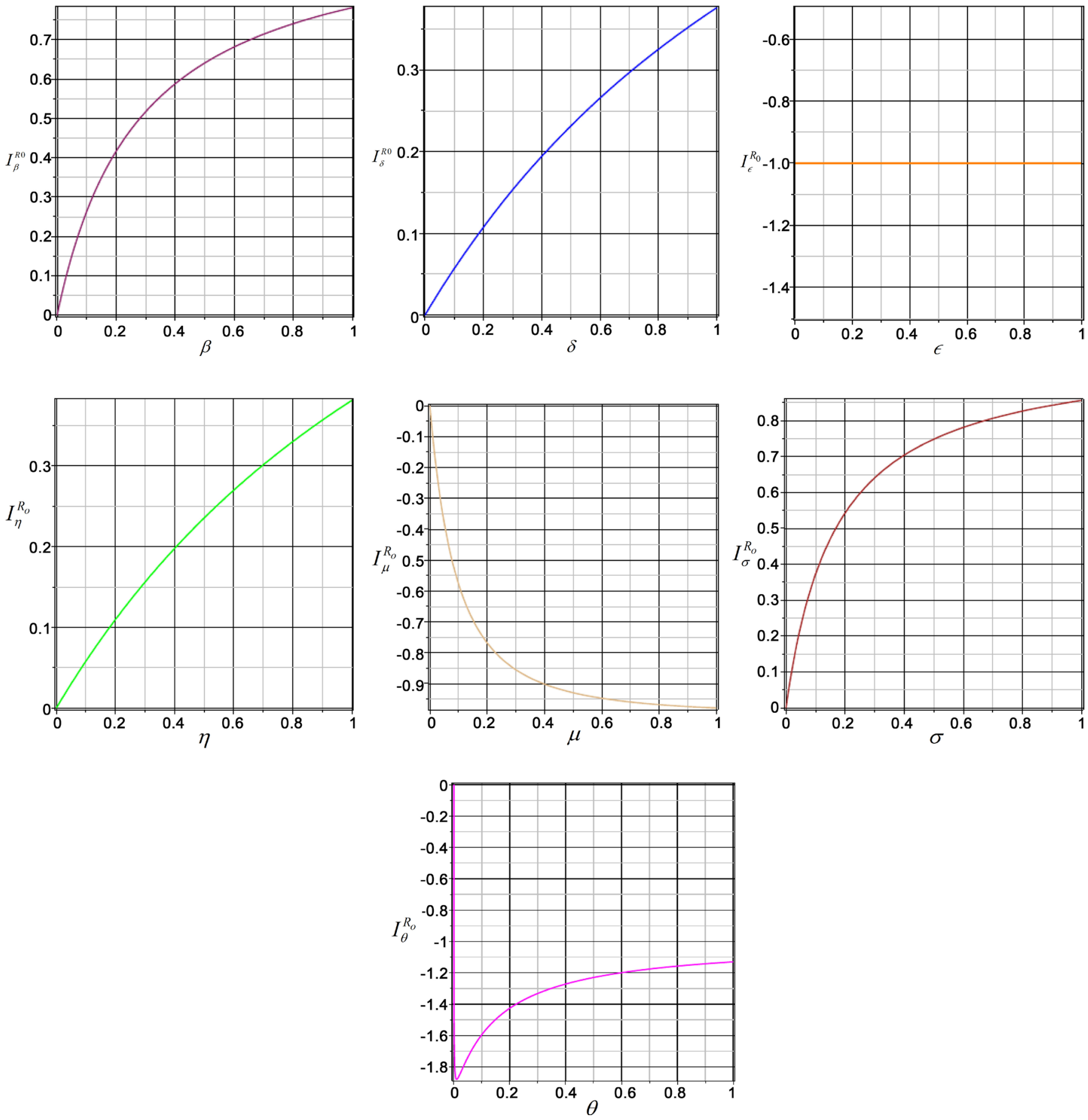

Figure 4. Behavior of the sensitivity indexes for $I_{\beta}^{R_{o}}, I_{\delta}^{R_{o}}, I_{\rho}^{R_{o}}, I_{\theta}^{R_{o}}, I_{\mu}^{R_{o}} y I_{\sigma}^{R_{o}}$.

to the most sensitive parameters.

In Figure 4, it is shown the behavior for each sensitivity index as function of each parameter.

\section{Simulations}

The simulations of system (1)-(3) were obtained using the values of the parameters as reported in Table 1 and the MAPLE software. 
From the results of the simulations, which are depicted in Figure 5 and Figure 6, it is show that the dynamic system, which describes the dynamics of the infectious process, stabilizes quickly in the first ten days for different values of the ZIKV transmission probability from the infected men to susceptible women, for small, medium and high values of probability. This is to analyze the behavior of the populations, see Table 3 . If we consider some preventive measures to decrease the probability to 0.3 , the stability level by sexual transmission decreases in almost an $84 \%$.

Figure 6 displayed the behavior of the human and mosquitoes populations for a probability of sexual transmission of 0.8 . These ones stabilize in the first ten days of the infectious process.

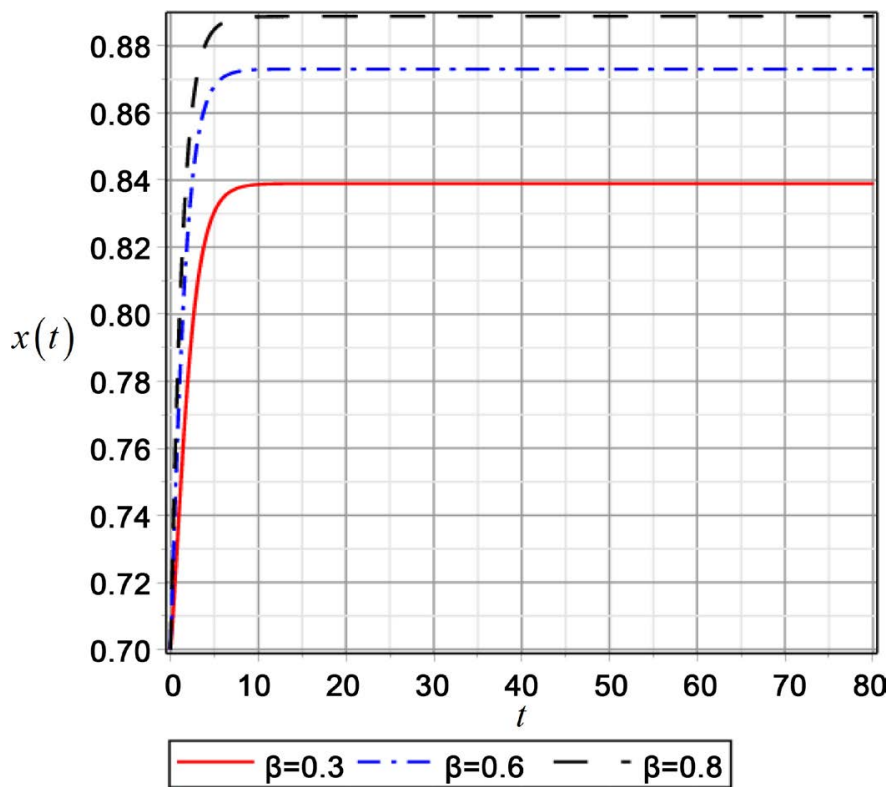

Figure 5. Behavior of the fraction of infected by ZIKV for different $\beta$.

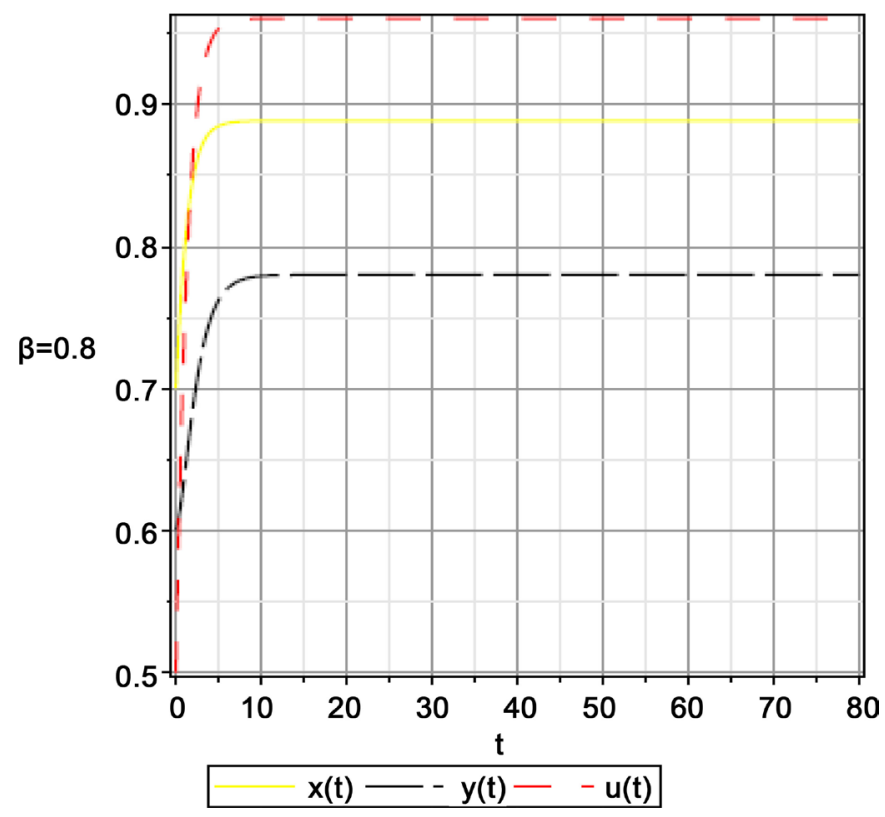

Figure 6. Human and mosquitoes population behavior for $\beta=0.8$. 


\section{Conclusions}

It is possible to see that upon beta increases, the infected women population increases in a time $t$. Then, it is extremely important to apply prevention measures in the sexual relationships of the infected population, and then help to stop the virus propagation at large scale. Together with these measures, it is expected to have a decrease in the diseases derived by this virus, as congenital microcephaly and Guillian-Barre syndrome.

Figure 6 shows the behavior of the infected women's fraction, infected men's fraction and virus-carrier mosquitoes fraction with a parameter $\beta=0.8$ from which it is reflected a great quantity of infected men and women as well as a considerable number of virus-carrier mosquitoes, from which, it is evidenced the importance that has the level of the virus transmission by sexual way.

The basic reproduction number is inversely proportional to the recovery rate of the infected persons and the mortality rate of the mosquitoes. That is to say, it is not appropriate to decrease the values of these parameters by treatment and control of the mosquito.

Actually, the Ross-Macdonald formalism is very important to model Vector-Host diseases as Malaria, Dengue, Chagas, Chikungunya and ZIKV, etc. In future works it is important to carry out the goblal stability analysis of the model and add the horizontal transmission in the man as well as the model without considering Sir. Ronald Ross formalism.

\section{Acknowledgements}

AML thanks to Grupo de Modelación Matemática en Epidemiología (GMME), Facultad de Educación, Vicerrectoría de investigaciones, Universidad del Quindío-Colombia.

\section{References}

[1] Heymann, D.L., Hodgson, A., Sall, A.A., Freedman, D.O., Staples, J.E., Althabe, F., Baruah, K., Mahmud, G., Kandun, N., Vasconcelos, P.F.C., Bino, S. and Menon, K.U. (2016) Virus Zika y microcefalia: Por qué esta situación es un ESPII. The Lancet, 387, 719-721. https://doi.org/10.1016/S0140-6736(16)00320-2

[2] Rodríguez-Morales, A.J. (2016) Zika y microcefalia en América Latina: ¿una amenaza emergente para los viajeros embarazadas? Viajes Med Infect Dis., 14, 5-6.

[3] Musso, D. and Baud, D. (2016) Zika Virus: Time to Move from Case Reports to Case Control. The Lancet Infectious Diseases, 16, 620-621. https://doi.org/10.1016/S1473-3099(16)00096-7

[4] European Centre for Disease Prevention and Control (2015) Zika Virus Epidemic in the Americas: Potential Association with Microcephaly and Guillain-Barre Syndrome. European Centre for Disease Prevention and Control, Stockholm.

[5] Musso, D., Roche, C., Nhan, T.-X., Robin, E., Teissier, A. and Cao-Lormeau, V.-M. (2015) Detection of Zika Virus in Saliva. Journal of Clinical Virology, 68, 53-55. https://doi.org/10.1016/j.jcv.2015.04.021

[6] Mansuy, J.M., Dutertre, M., Mengelle, C., Fourcade, C., Marchou, B., Delobel, P., et al. (2016) Zika Virus: High Infectious Viral Load in Semen, a New Sexually Transmitted Pathogen? The Lancet Infectious Diseases, 16, 405. https://doi.org/10.1016/S1473-3099(16)00138-9 
[7] Gourinat, A.C., O’Connor, O., Calvez, E., Goarant, C. and Dupont-Rouzeyrol, M. (2015) Detection of Zika Virus in Urine. Emerging Infectious Diseases, 21, 84-86. https://doi.org/10.3201/eid2101.140894

[8] Foy, B.D., Kobylinski, K.C., Chilson Foy, J.L., Blitvich, B.J., Travassos da Rosa, A., Haddow, A.D., Lanciotti, R.S. and Tesh, R.B. (2011) Probable Non-Vector-Borne Transmission of Zika Virus, Colorado, USA. Emerging Infectious Diseases, 17, 880-882. https://doi.org/10.3201/eid1705.101939

[9] Musso, D., Roche, C., Robin, E., Nhan, T., Teissier, A. and Cao-Lormeau, V.M. (2015) Potential Sexual Transmission of Zika Virus. Emerging Infectious Diseases, 21, 359-361. https://doi.org/10.3201/eid2102.141363

[10] Atkinson, B., et al. (2016) Detection of Zika Virus in Semen. Emerging Infectious Diseases, 22, 940. https://doi.org/10.3201/eid2205.160107

[11] Venturi, G., et al. (2016) An Autochthonous Case of Zika Due to Possible Sexual Transmission, Florence, Italy 2014. Eurosurveillance, 21, Article Number: 30148. https://doi.org/10.2807/1560-7917.ES.2016.21.8.30148

[12] Armstrong, P., Hennessey, M., Adams, M., et al. (2016) Travel-Associated Zika Virus Disease Cases among US Residents. Morbidity and Mortality Weekly Report, 65, 286-289. https://doi.org/10.15585/mmwr.mm6511e1

[13] Patiño-Barbosa, A.M., Medina, I., Gil-Restrepo, A.F. and Rodriguez-Morales, A.J. (2015) Zika: Another Sexually Transmitted Infection? Sexually Transmitted Infections, 91, 359. https://doi.org/10.1136/sextrans-2015-052189

[14] Instituto Nacional de Salud. Dirección de Vigilancia y Análisis del Riesgo en Salud Pública.

[15] Gao, D., Lou, Y., He, D., Porco, T.C., Kuang, Y., Chowell, G. and Ruan, S. (2016) Prevention and Control of Zika as a Mosquito-Borne and Sexually Transmitted Disease: A Mathematical Modeling Analysis. Scientific Reports, 6, Article Number: 28070.

[16] Pizza, D.M.M., Loaiza, A.M., Montoya, J.F.A., Manrique, O.A., Sossa, V.A., Muñoz, C.A. and Enríquez, M.A. (2016) A Model for the Risk of Microcephaly Induced by the Zika Virus (ZIKV). Open Journal of Modelling and Simulation, 4, 109-117. https://doi.org/10.4236/ojmsi.2016.43010

[17] Van, P. and Watmough, J. (2002) Reproduction Numbers and Sub-Threshold Endemic Equilibria for Compartmental Models of Disease Transmission. Mathematical Biosciences, 180, 29-48. https://doi.org/10.1016/S0025-5564(02)00108-6

[18] Holland, J. (2007) Notes on R0. Department of Anthropological Sciences, Stanford University, Stanford.

[19] Diekmann, O., Heesterbeek, J. and Roberts, M. (2010) The Construction of Next-Generation Matrices for Compartmental Epidemic Models. Journal of the Royal Society Interface, 7, 873-885. https://doi.org/10.1098/rsif.2009.0386

[20] Rodrigues, H., Monteiro, M. and Torres, D. (2013) Sensitivity Analysis in a Dengue Epidemiological Model. Conference Papers in Mathematics, 2013, Article ID: 721406.

[21] Chitnis, N., Hyman, J. and Cushing, J. (2008) Determining Important Parameters in the Spread of Malaria through the Sensitivity Analysis of a Mathematical Model. Bulletin of Mathematical Biology, 70, 1272-1296. https://doi.org/10.1007/s11538-008-9299-0

[22] Hamby, D. (1994) A Review of Techniques for Parameters Sensitivity Analysis of Environmental Models. Environmental Monitoring and Assessment, 32, 135-154. https://doi.org/10.1007/BF00547132 
Submit or recommend next manuscript to SCIRP and we will provide best service for you:

Accepting pre-submission inquiries through Email, Facebook, LinkedIn, Twitter, etc. A wide selection of journals (inclusive of 9 subjects, more than 200 journals) Providing 24-hour high-quality service

User-friendly online submission system

Fair and swift peer-review system

Efficient typesetting and proofreading procedure

Display of the result of downloads and visits, as well as the number of cited articles Maximum dissemination of your research work

Submit your manuscript at: http://papersubmission.scirp.org/

Or contact ojmsi@scirp.org 